\title{
DEVELOPMENT OF EXTREME REGULATOR OF SEPARATION MOISTURE FROM THE GAS STREAM
}

\author{
Heorhii Kulinchenko ${ }^{1}$, Petro Leontiev², Oleksii Drozdenko ${ }^{3}$ \\ ${ }^{1}$ Department of Computer Science, Section Computerized Control Systems, Sumy State University, Sumy, Ukraine \\ georgv@ukr.net \\ ORCID: https://orcid.org/0000-0002-8501-5636 \\ ${ }^{2}$ Department of Computer Science, Section Computerized Control Systems, Sumy State University, Sumy, Ukraine \\ petrlkj000@gmail.com \\ ORCID: https://orcid.org/0000-0002-9494-9078 \\ ${ }^{3}$ Sumy State University, Sumy, Ukraine \\ o.drozdenko@ksu.sumdu.edu.ua \\ ORCID: https://orcid.org/0000-0002-0047-739X
}

A R T ICLE INF O
Article history:
Received date 23.03 .2021
Accepted date 27.04 .2021
Published date 30.04 .2021
Section:
Control systems
D O I

$10.21303 / 2313-8416.2021 .001815$

KEYWORDS

actuator

transport lag

flow throttling

moisture entrainment

search for an extremum

dew point temperature

\section{ABS TRACT}

Object of study: Automation of control of technological parameters of the process of low-temperature separation (LTS) of moisture from a gas stream.

Investigated problem: Improving the efficiency of the separation process when preparing gas for transportation through the consumption network. Development of hardware and software tools that provide automation of the process of regulating flow parameters under conditions of action of time-varying disturbances.

Main scientific results: A criterion for controlling the LTS process of a gas flow is formulated. The difference in the dynamic characteristics of the processes occurring during the separation of moisture makes it possible to effectively use a two-circuit control system. The perspective of using extreme regulators (ER) of the process, which is characterized by time delays between the input of the object and its output, is substantiated. The use of ER ensures the stability of the control process under the influence of disturbances in the parameters of the gas flow.

In the MATLAB environment, an ER model has been developed, in which a step-type algorithm is used, which provides an increase in the efficiency of the LTS process in accordance with the selected criterion

The area of practical use of research results: The scope of use of the research results are automation objects that need continuous automatic tracking of the changing settings of the process parameters regulators. The studies carried out make it possible to use the proposed regulator in various technological installations for drying gas and air (with appropriate identification of the parameters of the object).

Innovative technological product: Based on the formed criterion for controlling the LTS process, which is aimed at increasing the efficiency of the LTS process of moisture from a gas flow, a microprocessor-based step-type ER has been developed. Preliminary tests of the regulator show that it can be implemented on commercially available program logic controllers on the market.

Scope of application of an innovative technological product: The considered ER can be used to construct control systems for oil and gas transportation processes, which require adaptation and optimization modes to flow parameters. In this case, the need for adaptation is dictated by changes in flow parameters over time.

(C) The Author(s) 2021. This is an open access article under the Creative Commons CC BY license

\section{Introduction}

The processes of natural gas transportation to the consumer are accompanied by losses associated with ensuring the quality indicators of the transported gas. One of the factors to reduce such losses is to improve the control of the low-temperature separation (LTS) process, which is carried out by throttling the flow and is used in the purification of natural gas. Finding a compromise between the achieved level of gas dehydration and the reduced productivity of a well or other source is an urgent task when optimizing the parameters of the LTS process. The peculiarities of the control object, which is the LTS installation, lead to the need to implement a complex structure of the regulator, which could provide maximum performance for the given parameters of the output stream. 
The problems associated with the compensation of disturbances in the gas flow parameters are classically solved by stabilizing the output parameters of the process. However, the ideology of passive stabilization does not provide for the adjustment of the regulator parameters when the parameters of the flow entering the throttling change over time. Respectively, the settings of the regulator, calculated for the optimum for the average moisture content of the flow, when its temperature or humidity changes, will lead to a loss of performance (efficiency) of the moisture separation process with any reconfiguration of the regulator. Therefore, to improve the efficiency of gas drying processes, hardware and software should be used to correct the settings of the separation process regulator in real time.

The objective of this research is to assess the possibilities of adaptive regulation of the parameters of the throttling process of the experimental LTS equipment, which would improve the efficiency of this process. The main factor in increasing efficiency is presented as a reduction in the time of transient processes when regulating the parameters of the gas flow throttling process.

\section{1. Object of research}

The object of research is the LTS process of moisture from a gas flow. A sufficient number of publications have been devoted to the development of hardware and software for regulating flow parameters under conditions of action of time-varying disturbances. Only a few of them investigate the issues of related control using adaptation methods when changing the parameters of the gas flow.

The subject of research is presented as an assessment of the possibilities of implementing a regulator that solves the problem of increasing the efficiency of the LTS process.

\section{2. Description of the problem}

The essence of the LTS process is described using the Joule-Thomson equation:

$$
T_{2}=T_{1}-\mu P_{D}
$$

where $T_{1}, T_{2}$ - temperature at the inlet and outlet of the unit; $P_{D}$ - pressure drop across the butterfly valve; $\mu$ - Joule-Thomson coefficient.

The control task is to change the outlet temperature of the stream $T_{2}$ so that this temperature is less than the dew point temperature (DPT) $T_{R}$ of the incoming stream. The result of separation is a decrease in humidity and DPT of the flow entering the consumer network. The DPT parameter serves as an indicator of the quality of the output stream, since the DPT indicates to what temperature the gas flow must be cooled in order to create conditions for the formation of dew.

The complexity of process control arises due to mutually contradictory requirements for the process: the higher the degree of product purification, the lower the productivity of the process, and vice versa. The dynamics of the process is determined by the rate of dew settling in the separator, and the settling rate has a finite value and depends on the size of the formed droplets. In this case, an increase in the gas flow rate contributes to the entrainment of distillate particles into the pipeline [1]. Therefore, in industry, the measure of the efficiency of gas dehydration is the measurement of the droplet entrainment of condensate. Reducing the level of droplet entrainment of water and heavy hydrocarbons from the gas stream makes it possible not only to meet the requirements for the transported stream, but also to increase the profitability of production due to additionally extracted condensate [2].

Despite ongoing research to assess the droplet entrainment, on the market there are no instruments that allow in an industrial environment to measure the efficiency of drying in a gas stream. Therefore, a real approach to assessing the efficiency of gas dehydration is a variant that allows one to indirectly evaluate the parameters of the LTS process and control them.

The approach to optimizing the modes of gas treatment units assumes [3]: increasing the dry gas yield; increasing the output of stable condensate; assessment of the balanced performance of the installation; reduction of technological losses.

Then the efficiency of the gas drying process $\mathrm{E}$ can be estimated by the ratio:

$$
E=Q\left(\chi_{1}-\chi_{2}\right)
$$

where $\chi_{1}, \chi_{2}-$ moisture content of the stream before and after drying; $Q$ - gas consumption. 
In this case, the flow rate (gas flow rate $Q$ ) is limited by the value of $Q_{\max }$, at which droplets of the separated moisture begin to drift into the consumers highway. The measure of the moisture content is the DPT of the flow at the inlet and outlet of the separator.

The use of estimate (2) makes it possible to optimize the separation mode based on measurements of the gas flow rate and the difference in moisture content at the inlet and outlet of the installation. In this case, the difference in moisture content is characterized by the difference between the corresponding DPT.

It should be noted that the control of the LTS process does not provide for direct regulation of the gas flow rate $Q$, since the main control task is to ensure the conditions for moisture condensation. The latter problem is solved by means of a differential pressure control circuit $P_{D}$, at the butterfly valve. Accordingly, the setpoint pressure $P_{D}$, which is necessary to obtain the required flow temperature corresponding to the DPT, will come from the external control loop. The model diagram of the LTS process regulator shown in Fig. 1 has two control loops.

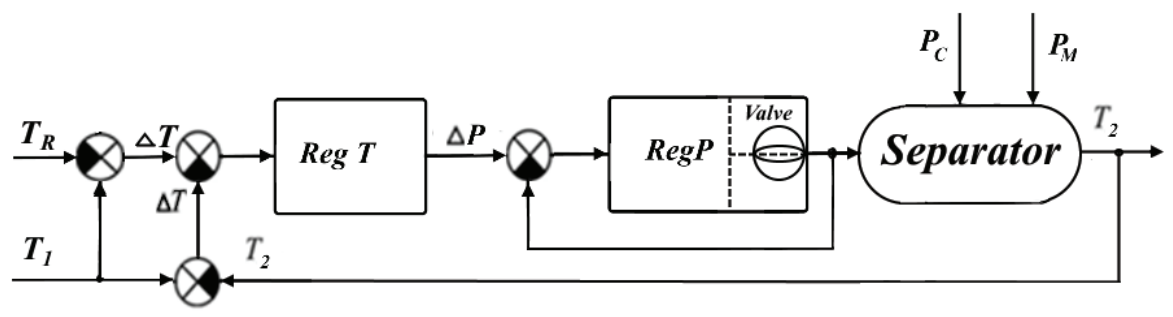

Fig. 1. Scheme of the model of the process regulator LTS

The need to introduce a second circuit is associated with the difference in the dynamics of the processes occurring in the separation unit. The first of them is the regulation of the gas flow pressure; the second is a change in the temperature of the flow due to its adiabatic expansion; the third is the condensation of moisture contained in the controlled flow.

Analysis of the LTS process as a control object [4] shows its following features:

- interconnections of parameters are described by nonlinear functions;

- the object is characterized by a transport delay due to the finite time of the flow and the settling time of the particles in the separator;

- process input variables: temperature, pressure and humidity change over time in a random way.

Additional difficulties in managing the object arise from the difference in the time constants of the processes taking place: pressure balancing, temperature exchange and moisture settling. In this case, the perturbations of the parameters of the input flow also differ in their dynamics.

In practical applications, in most cases, the construction of a control system is carried out on the basis of PID controllers. However, despite the simplicity of implementation and a significant number of theoretically substantiated methods for their adjustment, in the presence of delays of the control object, PID controllers do not always provide the required quality of regulation [5].

If the quality of regulation by our object is formulated as keeping the deviations of the controlled temperatures in a given range, then the implementation of the controller settings becomes difficult to implement. These difficulties are associated with the need to simultaneously compensate for the time delays of the parameters of the moisture condensation process and the dynamics of the control loops. Therefore, the settings of the regulator coefficients, obtained, for example, by the Ziegler-Nichols method, are approximate and need constant adjustment due to the nonstationarity of the flow parameters. But the possibilities of adjusting the parameters are limited by the stability conditions of the object by regulation.

As a result of the development of programming tools and modern microprocessor technology, it became possible to compensate for the response delay of an object on the basis of the Reisvik controller and the Smith predictor [6]. But their use gives positive results only if the parameters of the object and the model match exactly. Perturbations of the parameters of the object in time (more than $5 \%$ ) do not allow obtaining the given parameters of the quality of regulation. In this case, the regulators can become unstable. It is possible to improve these parameters by further complicating the hardware and software based on systems with adaptation or an optimization loop. 
The objective of research is presented as an assessment of the possibilities of implementing a regulator that is able to adapt to changes in the parameters of the gas separation process.

\section{3. Proposed way to solve the problem}

The control task is formulated as finding such control actions that provide an increase in the system efficiency in the presence of external disturbances. Extreme controllers, which, in terms of implementation complexity, occupy an intermediate position between systems with adaptation and PID controllers, are also used to control objects with delay [7, 8].

It should be noted that in the process of constructing an extremal controller, it is also necessary to solve problems associated with the difference in the dynamics of the reaction of the control object and the dynamics of the acting disturbances of the parameters. It is justified to state that perturbations in the gas flow temperature are characterized by slower changes than changes in the flow pressure. The experimental unit, which studies the regulator of the moisture separation process [9], makes it possible to evaluate the relationship between the dynamic characteristics of the separation process. The obtained estimates give grounds to form extreme control actions without calculating the response of the object model in each cycle, thereby increasing the speed of the process controller.

The aim of this research is to study the possibility of using an extreme regulator of the LTS process to increase the efficiency of this process.

The presence of disturbances in the parameters of the flow entering the separation imposes restrictions on the ranges of applicability of the controller settings obtained for specific operating points of the nonlinear plant. This circumstance leads to the need to assess the influence of errors associated with assumptions about the degree of influence of the method for describing the nonlinearity of an element on the result of calculating the process mode.

The study of options for the implementation of the LTS process control system involves the assessment of costs for the implementation of the developed software, first on an experimental plant, and in the future on industrial equipment.

\section{Materials and methods}

\section{1. The structure of the separation process control system}

In accordance with the scheme for modeling the control of the LTS process (Fig. 1), the structure of the regulator is formed by two control loops (RegP and RegT). The first circuit shown in Fig. 2, ensures the maintenance of the differential pressure required to ensure the condensation mode (set DPT).

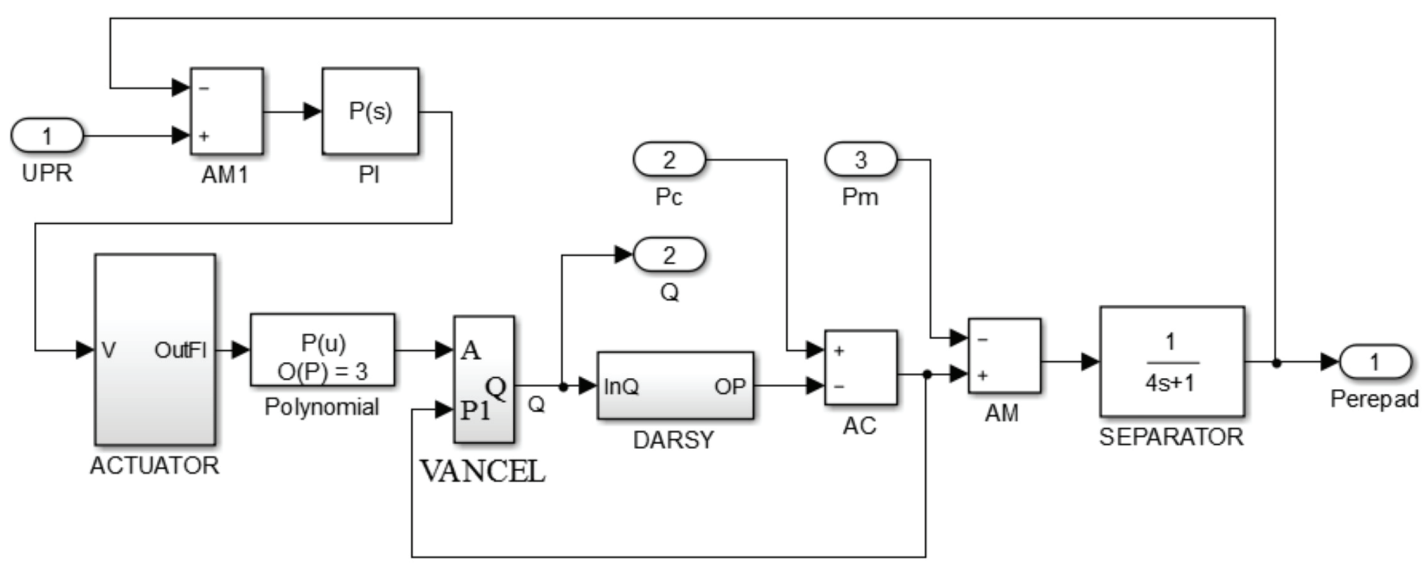

Fig. 2. Scheme of the control loop RegP

The second loop RegT, which is slower, is designed to correct the separation mode at the UPR input when the parameters of the inlet gas flow change. These two circuits control the position of the valve, which is driven by an Actuator at a constant travel speed.

In addition to the actuator, which in the simulation is represented by an integrator with a transfer coefficient $K_{I}$, the dynamics of the object is affected by the process of moisture deposi- 
tion in the separator. This process, when strictly examined, appears to be an aperiodic link with a delay. In our case, when a model of an experimental separation plant is considered, the dynamics of the process can be approximated by an aperiodic link with an equivalent time constant. The expediency of such an approximation is dictated by the desire to ensure the stability of the numerical simulation process. The reason for this step is the ratio of the response time and delay time constants.

As a result of the object linearization procedures carried out using the Matlab application "Linear Analysis Points" for each operating point, linearization, the transfer function of the closedloop differential pressure control is obtained $\mathrm{P}_{D}$ third order:

$$
W_{P D}(s)=\frac{b_{1 i} s+b_{0 i}}{s^{3}+a_{2 i} s^{2}+a_{1 i} s+a_{0 i}},
$$

where $i$ - set point number; $a_{i}, b_{i}$ - polynomial coefficients.

This set of linearized transfer functions corresponds to a family of PID controllers, which are also tuned using the Matlab application "SISO Design Tool".

Due to the nonlinear nature of the object under consideration and significant phase shifts in the second, external loop, it was not possible to implement stable control based on the PID controller. An acceptable version of the external loop regulator turned out to be an extreme regulator (ER), which corrects the preset value of the pressure drop as a result of evaluating the gas flow rate $Q$. This choice of the optimization criterion is explained by the fact that the process efficiency is the measure of the process efficiency. Accordingly, if the inner loop provides the condition for moisture condensation (lowering the flow temperature to the DPT), then the task of the outer loop is to ensure the maximum possible gas flow rate, but not higher than $Q_{\max }$, at which moisture is carried away from the separator.

In this variant of the control system structure, the ER loop is connected in parallel with the differential pressure regulator, which allows the valve position to be corrected when the parameters of the flow entering the separation are changed (Fig. 3).

The role of the disturbance simulator is played by the VarT1 generator, with the help of which disturbances of different frequencies and amplitudes are specified. The OBJect model subsystem includes the model blocks shown in Fig. 2 - Actuator gate valve "Actuator" and moisture settling unit "Separator".

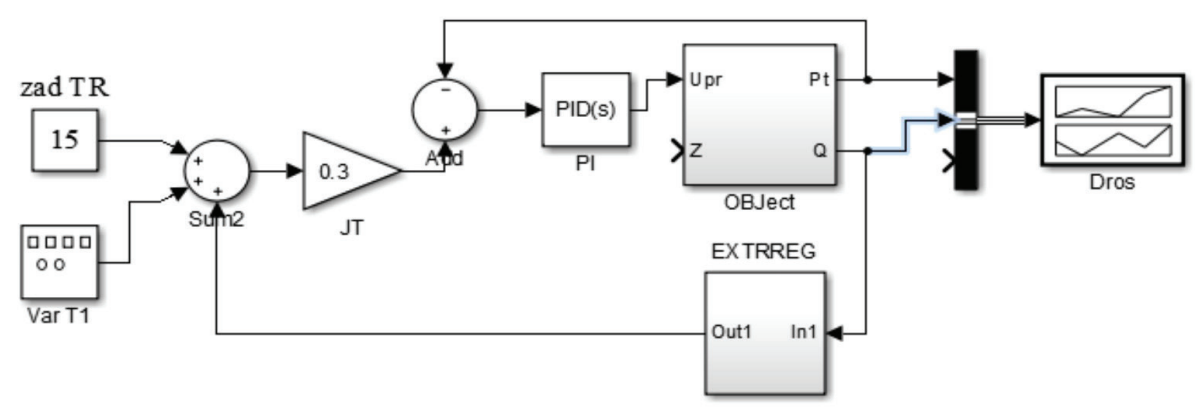

Fig. 3. Schematic of the model with an extreme regulator (EXTReg)

\subsection{Algorithm of the extreme regulator}

The implementation of the ER provides for the choice of an algorithm for its functioning, while the need to develop an original algorithm arises only in the presence of the specifics of the functioning of the object [10]. To develop an ER control algorithm, let's concretize the functions that are produced by the regulator:

- finding the extreme value of the flow rate in the control process;

- limitation of gas flow rate;

- reducing the time of the transient process when regulating.

The construction of the ER algorithm depends on the principle in accordance with which the direction of movement to the extremum of the selected criterion is determined. 
The following principles are widely used (depending on the requirements for cost and reliability):

- extremum memory regulators;

- step-type regulators, working out signs of adjustable value increments;

- derivative sign-responsive regulators.

Other well-known regulators, for example, of a mixed type, have a more complex structure and have not been considered at this stage.

The simplest to implement are controllers that respond to the sign of the derivative of the output signal to the input signal. In our object, which has large time delays, this operation loses its meaning due to the asynchronous measurement of the mentioned signals. The absence in such a regulator of an element that fixes the previous value of the criterion leads to inadequate regulation results.

Extremum memory controllers developed using MATLAB tools are widely used in various industries [11]. A characteristic feature of such regulators is that, thanks to the use of a signal relay, the ER fixes the reached extremum in a memory device. In the event of a decrease in the signal, the control action is not generated.

Step-type ERs have a greater degree of protection against perturbations. In such a regulator, at time intervals $\Delta t$, the controlled signal is measured. As a result of comparison of the controlled value, after each cycle $\Delta t$, a control action $U_{n+1}$ is generated, aimed at reaching an extremum. The algorithm for the formation of the control action is determined by the following relation:

$$
U_{n+1}=U_{n}^{*} G_{n+1}
$$

where $G_{n+1}=\operatorname{sign}(\Delta Q) \operatorname{sign} G_{n}$ - switching function; $n$ - time sampling step number; $\Delta Q$ - increment of the input value at the $n$-th step.

A schematic diagram of a step-type ER model, built according to algorithm (4), is shown in Fig. 4. Sampling of the signal in time is carried out by the Zero Order Hold element, memorization - using the delay element. As a result of comparison of the previous and current values of the controlled signal, a switching function is formed, which is fed to the ER output.

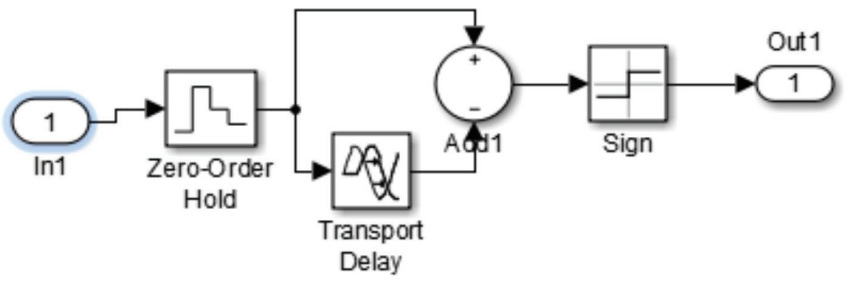

Fig. 4. Diagram of the extreme stepping type regulator

\section{Regulator operation simulation results}

To assess the functioning of the developed step-type ER, let's compare the oscillograms of the output signals obtained in the model circuit shown in Fig. 3. The simulation results are shown in Fig. 5.

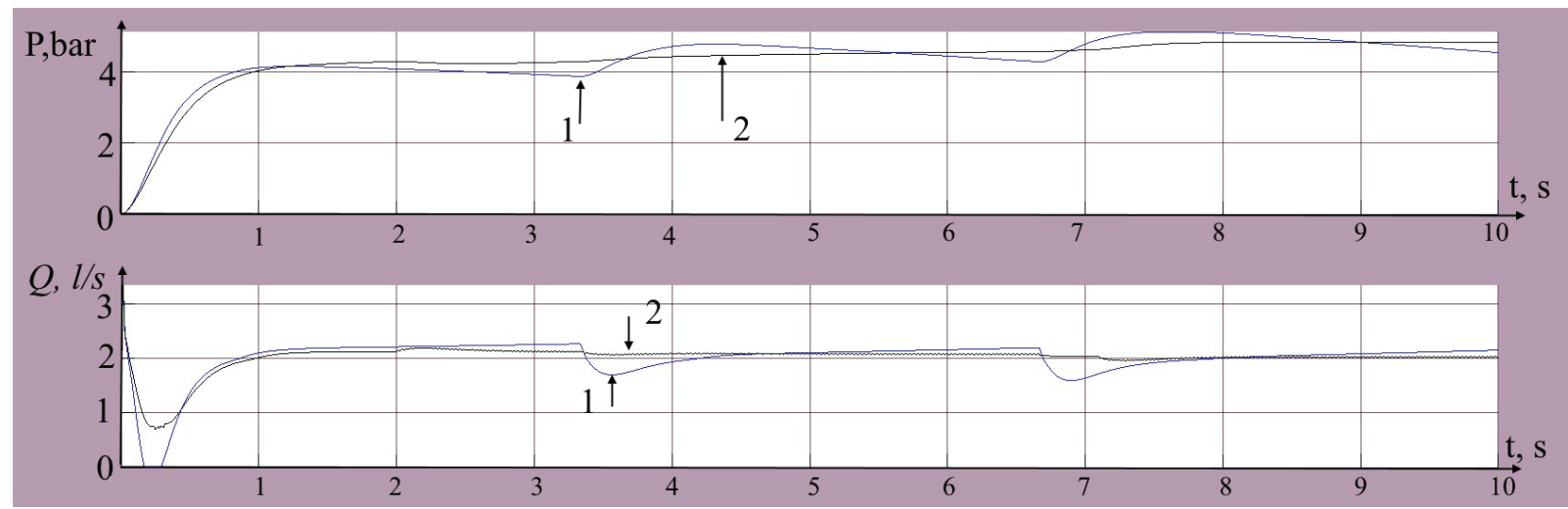

Fig. 5. Oscillograms of an object with off (1) and on (2) ER 
As can be seen from the oscillograms, the presence of an extreme regulator provides a decrease in the effect of disturbances in the NTS model, in which a gate valve drive of constant movement speed is used.

As a result of the action of the ER, there is also a stabilization of the pressure drop, which provides the required moisture condensation temperature. Minimization of dips in the gas flow rate over time under the action of disturbances, in accordance with the selected control criterion, makes it possible to increase the efficiency of the process.

The presence of some fluctuations in the signal trajectory when the ER is "on" indicates that the extremum value is in the zone and is being searched for. The level of oscillations can be adjusted by the ratio of the sampling time value and the delay time (storage of the previous signal sample), depending on the nature of fluctuations of the object parameters.

\section{Discussion}

The main advantage of the developed ER is the reduction of the complexity of regulation of the process parameters, which are typical for nonlinear objects. The use of ER made it possible to implement double-loop control, which is important in the context of conflicting requirements for the parameters of the LTS process.

The specificity of the dynamics of the moisture deposition process and the dynamics of the actuator during the implementation of the regulator requires accurate data on the object model and serious computational resources used for control. The proposed regulator is implemented in hardware on commercially available programmable logic controllers.

The search for optimal control modes allows many options for solving the problem, each of which leads to its own structure for constructing a regulator. In this work, let's consider simple versions of the ER. At the same time, the fundamental possibility of using such regulators for the process under study was assessed. Therefore, at this stage, the issues of drift of characteristics, boundaries of stability and improvement of the quality of regulation were not investigated.

As a result of the development of ER for objects of different nature [12], it is difficult to single out the significant advantages of one or another regulator. This is due to the fact that the method of searching for an extremum, the accuracy of tuning procedures that are successfully applied at one object, for example, when controlling fuel combustion [13], is inapplicable for the process of electrical discharge machining. Then, in accordance with the change in the object control tasks, the methods of combined control are used [14]. In the case of combined control, several tasks are solved: increasing productivity, performance and noise immunity. Studies carried out on models show the effectiveness of using such regulators: improved stability $-18 \%$, reduced energy consumption $-10 \%$, increased productivity $-20 \%$.

To evaluate the effectiveness of one or another type of regulator, separate studies are carried out [15], in which the conditions for conducting experiments are stipulated.

In our case, the ER design was carried out for the conditions of the operation of the experimental separation plant, focusing on the installed automation tools. Therefore, the simulation of disturbances and the ranges of changes in the parameters of the air flow only approximately reproduce the conditions of a real object of separation. However, the results obtained make it possible to formulate requirements for the modernization of the installation to improve the developed ER, which is included in the control system of the LTS process.

The efficiency of extreme regulation by the parameters of the process proceeding under the conditions of the action of random disturbances can be improved, for example, by using critical feedback [16]. The advantage of this approach lies in the simplicity of implementation, which follows from the extremum search algorithm. However, the effectiveness of such an algorithm in practical applications has not yet been confirmed.

Therefore, a real prospect for improving the ER is the development of an ER of the combined type.

\section{Conclusion}

Based on the formed criterion for controlling the LTS process, which provides for obtaining the maximum gas flow rate, in the presence of restrictions on the rate of sedimentation and moisture content, an ER model has been developed, which is based on an algorithm for searching for an 
extremum of a step type. The use of the proposed algorithm makes it possible to implement a twoloop control system for the LTS process with its simple hardware and software implementation.

The main advantage of ER, in comparison with PID controllers, in the regulation of moisture separation processes, is the ability to adapt to changing flow parameters. The solution of the problem of optimization of the LTS mode with the help of the ER makes it possible to increase the stability of the regulation process by reducing the overshoot value by $15 \%$. This reduces the regulation time by $25 \%$, which also leads to an increase in the productivity of the process.

The use of an algorithm with a stepwise search for an extremum makes it possible to implement a two-loop control system for the LTS process and to simplify the hardware-software implementation of the regulator.

The results of ER modeling show the possibilities of its improvement and the prospects of using it in experimental industrial applications.

\section{References}

[1] Wang, K., Ye, J., Bai, B. (2017). Entrained droplets in two-phase churn flow. Chemical Engineering Science, 164, $270-278$. doi: http://doi.org/10.1016/j.ces.2017.02.028

[2] Yarkeeva, N. R., Ishbulatov, I. A. (2019). To the question of low-temperature separation technology efficiency. Petroleum Engineering, 17 (1), 13-23. doi: http://doi.org/10.17122/ngdelo-2019-1-13-23

[3] Zobnin, A. A., Ivanov, S. S., Yiriakov, V. J. (2013) Optimizacia reiimov raboty ustanovok kompleksnoy podgotovki gaza. Problemy geologii i osvoeniia nedr. Tomsk, 2, 118-120.

[4] Kulinchenko, G. V., Pavlov, A. V., Leontiev, P. V. (2015). Forming the Approach to Designing the Controller of the Process of Low-temperature Natural Gas Separation. Bulletin of Vinnytsia Polytechnic Institute, 6, 9-17.

[5] Silva, G. J., Datta, A., Bhattacharyya, S. (2005) PID Controllers for Time-Delay Systems. Boston: Birkhauser, 329. doi: http:// doi.org/10.1007/b138796

[6] Mirzal, A. (2017). Delay Compensation using the Smith Predictor: A Brief Review with Numerical Examples. International Journal of Computer-Aided Mechanical Design and Implementation, 3 (1), 1-8. doi: http://doi.org/10.21742/ijcmdi.2017.3.1.01

[7] Zhang, C., Ordez, R. (2012). Extremum-Seeking Control and Applications. Springer-Verlag, 203. doi: http://doi.org/10.1007/ 978-1-4471-2224-1

[8] Burns, D. J., Weiss, W. K., Guay, M. (2015). Realtime setpoint optimization with time-varying extremum seeking for vapor compression systems. Proceedings of the 2015 American Control Conference (ACC). Chicago, 974-979. doi: http://doi.org/ 10.1109/acc.2015.7170860

[9] Kulinchenko, G. V., Leontiev, P. V. (2017) Solving Problems of Moisture Separation Based on SCADA Technologies. Bulletin of Vinnytsia Polytechnic Institute, 3, 14-23.

[10] Andreev, S. M., Sologubov, A. Yu., Parsunkin, B. N. (2016). Synthesis of seeking automatic optimization system for process combustion fuel. Russian Internet Journal of Industrial Engineering, 4 (1), 74-83. doi: http://doi.org/10.24892/rijie/20160110

[11] Rubanov, V. G., Bushuev, D. A. (2012). Simulation of extremum seeking control systems using Matlab and Simulink as mean of dynamics analysis// Nauchnye vedomosti BelGU, 19 (138), 24/1, 169-175.

[12] Nikolaev, A. B., Ostrouh, A. B., Marsov, V. I., Iliuhin, A. B. (2011). The comparative analysis of systems of extreme regulation of processes of transportation oil\&gas\&water mixes. Modern high technologies, 3, 35-39. Available at: http://www.top-technologies.ru/ru/article/view?id=26833

[13] Avdeeva, O. V. (2015). Systema ekstremalnogo regulirovania goreniem topliva v kotelnoi ustanovke. Vestnik Pensenskogo gosudarstvennogo universiteta, 3 (11), 167-174.

[14] Avdeeva, O. V., Artamonov, D. V., Nikitin, A. S., Semenov, A. D. (2013). Automation of electric erosion machining using the method of extreme combined control. University proceedings. Volga region Engineering sciences, 4 (28), 212-224.

[15] Zasyadvovk, O. V., Pysarenko, L. D. (2014). Synthesis of extreme control systems. Electronics and Communications, 19 (3 (80)), 99-105. doi: http://doi.org/10.20535/2312-1807.2014.19.3.141480

[16] Moroz, V., Turych, O., Marushchak, Ya. (2012). The new algorithm of the extremal control. Elektromehanichni i energozberigaiuchi systemy, 3 (19), 419-420. 